\title{
Symptomatic Steno-0cclusion in Patients with Acute Cerebral Infarction: Prevalence, Distribution, and Functional Outcome
}

\author{
Jihoon Kang, ${ }^{\mathrm{a}, \mathrm{l}}$ Tai Hwan Park, ${ }^{\mathrm{b}}$ Kyung Bok Lee, ${ }^{\mathrm{c}}$ Jong-Moo Park, ${ }^{\mathrm{d}}$ Youngchai Ko, ${ }^{\mathrm{e}}$ Soo Joo Lee, ${ }^{\mathrm{e}}$ \\ Keun-Sik Hong, ${ }^{\mathrm{f}}$ Yong-Jin Cho, ${ }^{\mathrm{f}} \mathrm{Ji}$ Sung Lee, ${ }_{\mathrm{g}}^{\mathrm{J}}$ Juneyoung Lee, ${ }^{\mathrm{h}}$ Byung-Chul Lee, ${ }^{\mathrm{i}}$ Kyung-Ho Yu, ${ }^{\mathrm{i}}$ \\ Dae-Hyun Kim, Jae-Kwan Cha, Jun Lee, ${ }^{\mathrm{k}}$ Myung Suk Jang, ${ }^{\mathrm{j}}$ Moon-Ku Han, ${ }^{\mathrm{a}}$ Hee-Joon Bae ${ }^{\mathrm{a}}$ \\ ${ }^{a}$ Department of Neurology, Cerebrovascular Center, Seoul National University Bundang Hospital, Seoul National University, Seongnam, Korea \\ ${ }^{b}$ Department of Neurology, Seoul Medical Center, Seoul, Korea \\ 'Department of Neurology, Soonchunhyang University College of Medicine, Seoul, Korea \\ ${ }^{\mathrm{d} D e p a r t m e n t ~ o f ~ N e u r o l o g y, ~ E u l j i ~ G e n e r a l ~ H o s p i t a l, ~ E u l j i ~ U n i v e r s i t y, ~ S e o u l, ~ K o r e a ~}$ \\ eDepartment of Neurology, Eulji University Hospital, Eulji University, Daejeon, Korea \\ ${ }^{\mathrm{f}}$ Department of Neurology, Ilsan Paik Hospital, Inje University, Koyang, Korea \\ ${ }^{g}$ Department of Biostatistics, Soonchunhyang University College of Medicine, Seoul, Korea \\ ${ }^{\mathrm{h}}$ Department of Biostatistics, Korea University College of Medicine, Seoul, Korea \\ IDepartment of Neurology, Hallym University College of Medicine, Anyang, Korea \\ 'Department of Neurology, Dong-A University College of Medicine, Busan, Korea \\ ${ }^{k}$ Department of Neurology, Yeungnam University Medical Center, Daegu, Korea \\ 'Department of Neurology, Samsung Changwon Medical Center, Sungkyunkwan University School of Medicine, Changwon, Korea
}

Background and Purpose Symptomatic steno-occlusion (SYSO) in acute ischemic stroke has a significant impact on treatment options and prognosis. However, the prevalence, distribution, clinical characteristics, and outcome of SYSO are not well known.

Methods We retrospectively identified 3,451 patients hospitalized because of ischemic stroke within 24 hours of symptom onset at 9 stroke centers in South Korea. Patients who did not undergo magnetic resonance imaging were excluded. SYSO was defined as stenosis or occlusion of cerebral arteries with relevant ischemic lesions in the corresponding arterial territory. The number, location, and severity of SYSOs and their effects on functional outcome were analyzed.

Results In total, 1,929 of 3,057 subjects (63.1\%) had SYSO. The most frequently affected vessels were the middle cerebral artery (34.6\%), extracranial internal carotid artery (14\%), vertebral artery $(12.4 \%)$, and basilar artery $(8.7 \%)$. SYSO predicted poor outcome on the modified Rankin Scale 3-6 (odds ratio, 1.77; 95\% confidence interval, 1.46-2.15) with adjustments. Involvement of 2 or more vessels was observed in 30.6\% of patients with SYSO and independently increased the risk of poor outcome (odds ratio, 2.76; 95\% confidence interval, 2.12-3.59). The severity of SYSO was associated with outcome and showed a significant dose-response trend $(P<0.001)$. The effect of SYSO on outcome did not significantly differ by individual arterial location ( $P$ for contrast $=0.21$ ).

Conclusions Approximately 60\% of patients with acute ischemic stroke had SYSO, and the severity and number were inversely correlated with outcome. The results suggest that SYSO could predict stroke outcome.
Correspondence: Hee-Joon Bae Department of Neurology, Stroke Center, Seoul National University Bundang Hospital, Seoul National University College of Medicine, 166 Gumi-ro, Bundang-gu, Seongnam 463-707, Korea Tel: +82-31-787-7467

Fax: +82-31-787-4059

E-mail: braindoc@snu.ac.kr

Received: December 10, 2013

Revised: December 23, 2013

Accepted: January 13, 2014

This study was supported by a grant from the Korea Healthcare Technology REtD Project, Ministry of Health and Welfare, Republic of Korea (HI10C2020).

The authors have no financial conflicts of interest.

Keywords Cerebrovascular occlusion; Stroke; Cerebral arteries; Magnetic Resonance Imaging

Copyright (C) 2014 Korean Stroke Society

This is an Open Access article distributed under the terms of the Creative Commons Attribution Non-Commercial License (http://creativecommons.org/licenses/by-nc/3.0/) which permits unrestricted non-commercial use, distribution, and reproduction in any medium, provided the original work is properly cited. 


\section{Introduction}

Evidence suggests that symptomatic steno-occlusion (SYSO) in acute ischemic stroke is associated with initial neurologic severity ${ }^{1}$ and stroke outcome. ${ }^{2-4}$ For the treatment of acute stroke, SYSO is not only the conceptual therapeutic target of intravenous thrombolysis ${ }^{5}$ but also the main target of intra-arterial thrombolysis, ${ }^{6}$ emergency endarterectomy, ${ }^{7}$ stent placement, ${ }^{8-10}$ and bypass operation.

With an increased focus on SYSO, several studies have attempted to elucidate the impact of SYSO on stroke outcome. These studies detected SYSO in 30\%-80\% of patients with ischemic stroke, ${ }^{1,11,12}$ and its location and severity were related to mortality, functional outcome, and stroke recurrence. ${ }^{13-15}$ However, a widely variable detection rate and inconsistent findings regarding the effect of the location and severity of SYSO has prohibited translation of these important findings to the general stroke population. For example, a variable time interval from stroke onset to study inclusion and the use of different diagnostic modalities may provide inconsistent results. We hypothesized that therapeutic or spontaneous recanalization of the acute occlusion vessel and discrepancies among diagnostic methods in detecting the vascular pathology may also contribute to heterogeneous findings. ${ }^{16-18}$ In addition, studies that allow enrollment of only selected cases and are conducted at a single center inhibit the generalization of findings.

In this study, we used a nationwide multicenter stroke registry to investigate the prevalence and distribution of SYSO in patients with acute ischemic stroke within 24 hours of stroke onset. ${ }^{19}$ To allow for feasible and accurate results, magnetic resonance angiography (MRA) was used for the vascular workup. The distribution of SYSO was characterized by location, number, and severity. In addition, we evaluated the contribution of SYSO and its characteristics to functional outcome.

\section{Methods}

\section{Study subjects and data acquisition}

In this retrospective observational study, we enrolled a consecutive series of 5,393 patients into the Clinical Research Center for Stroke program; these patients were admitted to one of 9 nationwide university or community-based hospitals in the Republic of Korea between September 2008 and March 2010 because of acute ischemic stroke. The Clinical Research Center for Stroke program was designed to prospectively collect clinical data from patients who experienced a stroke and to build a web-based multicenter stroke registry. The Clinical Research Center for Stroke plans to investigate the clinical evidence in the field of stroke prevention, management, and prognosis; to provide practical information; and to eventually aid decision making based on evidence. The registry also includes information on management performance measures as part of a quality improvement program. Each participating hospital received human research approval to enroll cases without individual patient consent under a common rule as well as exemption from subsequent review by their institutional review boards.

In total, 3,451 (64.0\%) of the enrolled patients who visited the hospital within 24 hours after stroke onset were included. We excluded 371 patients (10.8\%) who did not undergo diffusion-weighted imaging or cerebrovascular workup by MRA to evaluate the symptomatic lesion. After further excluding 23 patients who did not undergo assessment of functional outcome at discharge, 3,057 patients were included in the study.

We extracted the following data from the registry: demographic profile, medical history, smoking, detected risk factors, baseline stroke severity assessed by the National Institutes of Health Stroke Scale (NIHSS) score, stroke subtype by Trial of Org 10172 in Acute Stroke Treatment (TOAST) classification, thrombolysis, imaging findings, and functional outcome at discharge. A trained physician performed routine assessment of functional outcome with the modified Rankin Disability Scale (mRS) at discharge or transfer to a rehabilitation unit. Scores were categorized as good (mRS, 0-2) or poor (mRS, 3-6) outcome.

\section{Cerebral vasculature workup}

All images were obtained using whole body magnetic resonance imaging. Two-dimensional time-of-flight MRA was used for angiography. The imaging study was conducted on the day of admission, and the procedure was performed using the stroke protocol of each participating hospital.

Steno-occlusion of a cerebral artery was identified by the onsite neuroradiologist and reviewed by a neurologist. The stenosis was defined as a reduction in diameter or transient signal void with distal flow and categorized as either a mild $(<50 \%)$ or a moderate to severe ( $\geq 50 \%$ ) stenosis with some degree of modification. ${ }^{20}$ The occlusion denoted the loss of signal without distal flow. The investigated vessels were divided into 7 groups according to the location of the arterial lesion: extracranial internal carotid artery (EICA), intracranial internal carotid artery, middle cerebral artery (MCA), anterior cerebral artery, posterior cerebral artery, basilar artery (BA), and vertebral artery (VA). Ischemic lesions on diffusion-weighted magnetic resonance imaging with compatible clinical findings were classified according to the distribution of arterial territory. SYSO was diagnosed when stenosis or occlusion of the cerebral artery was observed 
with a relevant ischemic lesion. The number of SYSOs was categorized as the involvement of a single vessel or multiple vessels $(\geq 2)$. Involvement of multiple vessels was categorized as ipsilateral multiple anterior circulation, multiple posterior circulation, bilateral anterior circulation, and both anterior and posterior circulation. For involvement of a single vessel, the severity of SYSO was categorized as mild, moderate to severe, and occlusion. Location was investigated by 2 methods: involvement of the anterior versus posterior circulation and individual arterial location.

\section{Statistical analysis}

For univariate statistical analysis, $\chi^{2}$ test and Student $t$ test were used to study demographic characteristics and clinical variables and to study the relationship of variables with functional outcome. We chose the variables that were associated with the presence of SYSO $(P<0.2)$ for covariates of the multivariate model and considered the clinical plausibility.

The $\chi^{2}$ test and Student $t$ test were used to analyze the relationship between functional outcome and SYSO characteristics, including existence, number, severity, and location. Multivariate logistic regression models were developed to measure the adjusted odds ratios (ORs) of SYSO and the characteristics of poor functional outcome. For comparison of effects among each group on the basis of the number and location of SYSOs, we applied a contrast method using a combination of estimated coefficients from the logistic model. For evaluation of the severity of SYSO, a likelihood ratio test was applied to compare the dose-response trends with the outcome. Statistical significance was defined as $P<0.05$. All statistical analyses were performed using SPSS version 17.0 software (Chicago, IL, USA).

\section{Results}

\section{Baseline characteristics of study subjects}

The mean age of the 3,057 study subjects was $67.3 \pm 12.5$ years (male, $58.9 \%$ ), and their median baseline NIHSS score was 4 (interquartile range, $2-10$ ). In total, $15.1 \%$ of these subjects received thrombolytic therapy. At discharge, 1,401 subjects (45.8\%) were assessed as having a poor functional outcome. Poor outcome was associated with age, sex, diabetes, high risk of a cardioembolic source, smoking, history of stroke, baseline NIHSS score, and thrombolysis $(P<0.2)$ (Supplementary Table 1$)$.

\section{Distribution and clinical properties of SYSO}

A total of 1,929 subjects (63.1\%) had SYSO with more than mild stenosis. The detection rate of SYSO from stroke onset to hospital arrival within 3 hours, 6 hours, 12 hours, and 24 hours was $66 \%, 65.1 \%, 60.2 \%$, and $58.0 \%$, respectively.

A comparison of the baseline characteristics of the study subjects based on the presence of SYSO is shown in Table 1. Patients with SYSO were older and had significantly higher proportions of diabetes, high risk of a cardioembolic source, history of stroke, and more severe baseline NIHSS score. In total, 50.8\% of strokes were attributable to large artery disease and $25.6 \%$ were attributable to cardioembolic stroke.

Table 1. Baseline characteristics and acute stage treatment methods of study subjects

\begin{tabular}{|c|c|c|c|c|}
\hline & \multirow{2}{*}{$\begin{array}{l}\text { No symptomatic steno-occlusion (SYSO) } \\
\qquad(\mathrm{n}=1,128)\end{array}$} & \multirow{2}{*}{$\begin{array}{c}\text { SYSO } \\
(n=1,929)\end{array}$} & \multicolumn{2}{|c|}{ SYSO } \\
\hline & & & Single $(n=1,339)$ & Multiple $(n=590)$ \\
\hline Age (yr) & $64.9 \pm 13.0$ & $68.6 \pm 12.0^{\dagger}$ & $68.2 \pm 12.5$ & $69.6 \pm 10.9^{\dagger}$ \\
\hline Male & $683(60.5)$ & $1,118(58.0)$ & $775(57.9)$ & $343(58.1)$ \\
\hline Hypertension & $722(64.0)$ & $1,293(67.0)$ & $881(65.8)$ & 412 (69.8) \\
\hline Diabetes & $312(27.7)$ & $600(31.1)^{\ddagger}$ & $418(31.2)$ & $182(30.8)$ \\
\hline Dyslipidemia & $240(21.3)$ & $416(21.6)$ & $282(21.1)$ & $134(22.7)$ \\
\hline High risk of cardioembolism & $220(19.5)$ & $453(23.5)^{\ddagger}$ & $327(24.4)$ & $126(21.4)$ \\
\hline Smoking & $460(40.8)$ & $726(37.6)$ & $502(37.5)$ & $224(38.0)$ \\
\hline Stroke history & $213(18.3)$ & $442(22.9)^{\ddagger}$ & $292(21.8)$ & $150(25.4)$ \\
\hline $\begin{array}{l}\text { Initial National Institutes of Health Stroke Scale score, median } \\
\text { (interquartile range) }\end{array}$ & $3(1-6)$ & $6(2-14)^{\ddagger}$ & $5(2-13)$ & $8(3-16)^{\ddagger}$ \\
\hline \multicolumn{5}{|l|}{ Stroke subtype } \\
\hline Small vessel disease & $507(44.9)$ & $148(7.7)$ & $105(7.8)$ & $43(7.3)$ \\
\hline Large artery disease & $103(9.1)$ & $979(50.8)$ & $662(49.4)$ & $317(53.7)$ \\
\hline Cardioembolism & $274(24.3)$ & $494(25.6)$ & $356(26.6)$ & $138(23.4)$ \\
\hline Thrombolysis & $105(9.3)$ & $356(18.5)^{\ddagger}$ & $251(18.7)$ & $105(17.8)$ \\
\hline Intra-arterial thrombolysis & $3(0.3)$ & $123(6.4)^{\ddagger}$ & $79(5.9)$ & $44(7.5)$ \\
\hline Intra-arterial and intravenous thrombolysis & $1(0.1)$ & $73(2.4)^{\ddagger}$ & $53(4.0)$ & $20(3.4)$ \\
\hline
\end{tabular}

Values are presented as number (percent) unless otherwise noted. The relationships between no SYSO or SYSO and single or multiple SYSOs were analyzed separately. ${ }^{\dagger}$ and ${ }^{\ddagger}$ indicate $P<0.05$ by Student $t$ test and $\chi^{2}$ test, respectively. 
In patients with a single SYSO $(n=1,339)$, MCA was the most affected vessel, followed by EICA, VA, and BA (Figure 1). Of the single SYSOs, $70.6 \%$ were located in anterior vessels. The characteristics of old age, female sex, and high risk of a cardioembolic source were significantly greater in those with an anterior vessel SYSO compared with a posterior vessel SYSO.

In terms of severity, $25.5 \%$ of patients with a single SYSO had mild stenosis, $36.1 \%$ had moderate to severe stenosis, and

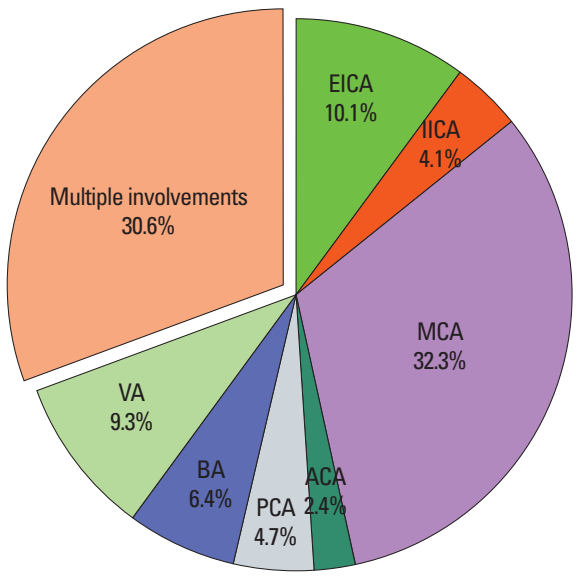

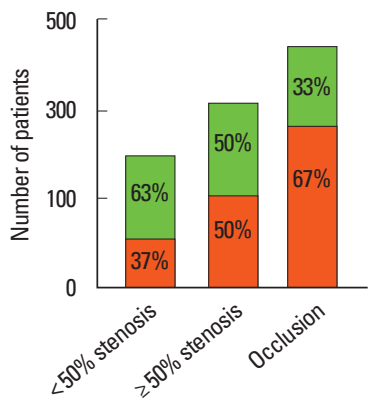

MCA

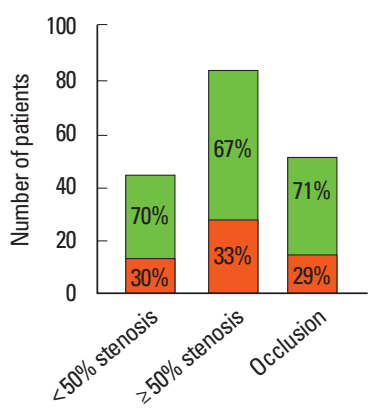

Vertebral artery

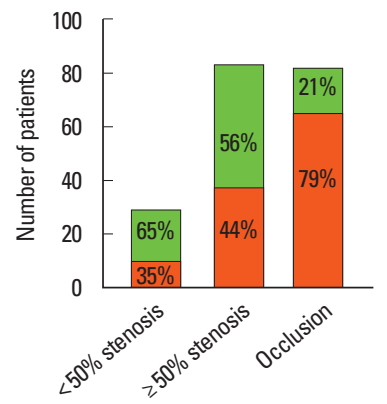

Extracranial ICA

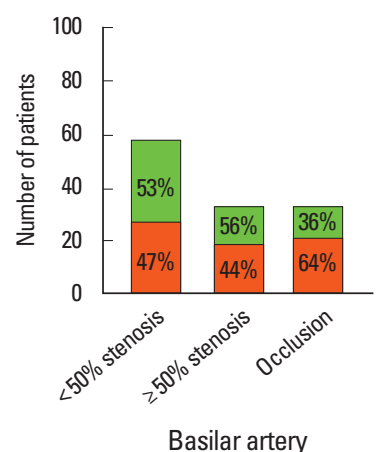

Figure 1. Distributed pattern of SYSO at the acute stage of ischemic stroke and relationship to functional outcome. The pie graph in the upper panel shows the distribution of single SYSO $(n=1,339)$. MCA was the most affected vessel, followed by EICA, VA, and BA. The bar graphs in the lower panel show the degree of steno-occlusion (x-axis) and functional outcome (y-axis). Outcome was assessed by mRS at discharge and categorized as good (blue; mRS, 0-2) or poor (green; mRS, 3-6). BA, basilar artery; EICA, extracranial internal carotid artery; IICA, intracranial internal carotid artery; MCA, middle cerebral artery; mRS, modified Rankin Disability Scale; SYSO, symptomatic steno-occlusion; VA, vertebral artery.
38.4\% had occlusion. As shown in Figure 1, symptomatic arterial occlusion was frequently observed in the MCA and EICA and moderate to severe stenosis was most frequently observed in the BA.

A total of 590 subjects (30.6\%) with SYSO had involvement of multiple vessels. Compared with patients with a single SYSO, involvement of multiple vessels was related to old age and a more severe baseline NIHSS score but not with high risk of a cardioembolic source. Among the patients with multiple SYSOs, involvement of both the anterior and posterior circulation (44.4\%) was the most commonly observed pattern, followed by involvement of the ipsilateral multiple anterior circulation (34.6\%), multiple posterior circulation (13.7\%), and bilateral anterior circulation $(7.3 \%)$.

\section{Impact of SYSO on functional outcome}

In total, $55.3 \%$ of patients with SYSO had a poor functional outcome, which was almost twice as high as that of patients without SYSO (Table 2). The multiple logistic model confirmed that the presence of SYSO was independently associated with a poor outcome at discharge (adjusted OR, 1.77; 95\% confidence interval [CI], 1.46-2.15) (Table 2 and Supplementary Table 2).

When the involvement of single and multiple vessels was analyzed separately in the multiple logistic model, both single SYSO (adjusted OR, 1.49; 95\% CI, 1.22-1.83) and multiple SYSOs (adjusted OR, 2.71; 95\% CI, 2.09-3.53) increased the risk of a poor outcome (Table 2). Additional analysis of the involvement of single and multiple vessels showed that the impact on functional outcome differed depending on the number of SYSOs $(P$ for contrast <0.001).

Analysis of the relationship between outcome and anterior versus posterior location of SYSO revealed that a poor outcome was more prevalent when SYSO was in the anterior circulation than in the posterior circulation (55.2\% vs. $37.1 \%)$. For individual arterial locations, the EICA, intracranial internal carotid artery, MCA, and BA showed a statistically significant crude OR for functional outcome (Table 2). However, the effect of the location on outcome disappeared in the multivariable logistic model $(P$ for contrast $=0.52)$, and an additional regression model introducing the individual arterial location showed similar results ( $P$ for contrast $=0.21)$.

Analysis of the relationship between the severity of a single SYSO and outcome showed that a moderate to severe degree of stenosis (OR, 1.41; 95\% CI, 1.07-1.85) and occlusion (OR, 2.00; $95 \% \mathrm{CI}, 1.51-2.65)$ of the cerebral vasculature significantly increased the risk of poor outcome compared with those without SYSO in the multivariable model. In addition, there was a significant dose-response relationship $(P$ for trend $<0.001)$. There 
Table 2. Impact of symptomatic steno-occlusion (SYSO) on poor functional outcome at discharge by multivariate analysis

\begin{tabular}{|c|c|c|c|c|}
\hline Variables & Good outcome (\%) & Poor outcome (\%) & $\begin{array}{c}\text { Crude odds ratio } \\
\text { (95\% confidence interval) }\end{array}$ & $\begin{array}{c}\text { Adjusted odds ratio } \\
\text { (95\% confidence interval) }\end{array}$ \\
\hline \multicolumn{5}{|l|}{ Presence of SYSO } \\
\hline No SYSO $(n=1,128)$ & $794(70.4)$ & $334(29.6)$ & 1 & 1 \\
\hline SYSO (n=1,929) & $862(44.7)$ & $1067(55.3)$ & $2.94(2.52-3.44)$ & $1.77(1.46-2.15)$ \\
\hline \multicolumn{5}{|l|}{ Number of SYSOs } \\
\hline None $(n=1,128)$ & $794(70.4)$ & $334(29.6)$ & 1 & 1 \\
\hline One $(n=1,339)$ & $671(50.1)$ & $668(49.9)$ & $2.37(2.00-2.80)$ & $1.50(1.22-1.84)$ \\
\hline Two or more $(n=590)$ & $191(32.4)$ & $399(67.6)$ & $4.97(4.01-6.16)$ & $2.76(2.12-3.59)$ \\
\hline \multicolumn{5}{|l|}{ Pfor contrast $<0.001^{\dagger}$} \\
\hline \multicolumn{5}{|l|}{ Location of single SYSO } \\
\hline No SYSO & $794(70.4)$ & $334(29.6)$ & 1 & 1 \\
\hline Anterior circulation ( $n=945)$ & $423(44.8)$ & $522(55.2)$ & $2.93(2.45-3.52)$ & $1.52(1.21-1.90)$ \\
\hline Posterior circulation ( $n=394)$ & $248(62.9)$ & $146(37.1)$ & $1.40(1.10-1.78)$ & $1.37(1.02-1.84)$ \\
\hline \multicolumn{5}{|c|}{ Detailed arterial location of single SYSO } \\
\hline No SYSO & $794(70.4)$ & $334(29.6)$ & 1 & 1 \\
\hline Extracranial internal carotid artery & $83(42.6)$ & $112(57.4)$ & $3.21(2.35-4.38)$ & $1.77(1.20-2.61)$ \\
\hline Intracranial internal carotid artery & $27(34.2)$ & $52(65.8)$ & $4.58(2.83-7.42)$ & $1.88(1.01-3.50)$ \\
\hline Middle cerebral artery & $284(45.5)$ & $340(54.5)$ & $2.85(2.32-3.49)$ & $1.42(1.10-1.84)$ \\
\hline Anterior cerebral artery & $29(61.7)$ & $18(38.3)$ & $1.48(0.81-2.69)$ & $1.41(0.65-3.03)$ \\
\hline Posterior cerebral artery & $67(73.6)$ & $24(26.4)$ & $0.85(0.53-1.38)$ & $0.76(0.43-1.35)$ \\
\hline Basilar artery & $58(46.8)$ & $66(53.2)$ & $2.71(1.86-3.94)$ & $1.88(1.19-2.97)$ \\
\hline Vertebral artery & $123(68.7)$ & $56(31.3)$ & $1.08(0.77-1.52)$ & $1.45(0.96-2.20)$ \\
\hline$P$ for contrast $=0.21^{\dagger}$ & & & & \\
\hline \multicolumn{5}{|l|}{ Severity of single SYSO } \\
\hline No SYSO (n=1,128) & $794(70.4)$ & $334(29.6)$ & 1 & 1 \\
\hline$<50 \%$ of stenosis $(n=342)$ & $221(64.6)$ & $121(35.4)$ & $1.30(1.01-1.68)$ & $1.07(0.79-1.45)$ \\
\hline$\geq 50 \%$ of stenosis $(n=483)$ & $262(54.2)$ & $221(45.8)$ & $2.01(1.61-2.50)$ & $1.41(1.07-1.85)$ \\
\hline Occlusion $(n=514)$ & $188(38.6)$ & $326(63.4)$ & $4.12(3.31-5.14)$ & $2.00(1.51-2.65)$ \\
\hline \multicolumn{5}{|l|}{$\chi^{2}$ for trend $=23.36, P<0.001^{\ddagger}$} \\
\hline
\end{tabular}

Each characteristic variable of SYSO adopted the multivariable logistic model. The multivariate model used the covariates of age, sex, history of stroke, initial National Institutes of Health Stroke Scale score, diabetes mellitus, high risk of a cardioembolic source, smoking, and thrombolysis. ${ }^{\dagger}$ and $^{\ddagger}$ indicate that $P$ values were obtained using a linear combination of estimated coefficients from the logistic model and the likelihood ratio test, respectively. The anterior circulation was composed of the anterior cerebral artery, middle cerebral artery, and intracranial internal carotid artery, and posterior circulation was composed of the posterior cerebral artery, basilar artery, and vertebral artery.

was no additional effect of severity and location of a single SYSO $(P$ for interaction effect $=0.45)$.

\section{Discussion}

In this study, $63 \%$ of patients with acute ischemic stroke had an MRA-defined steno-occlusion with a relevant ischemic lesion that independently affected the prognosis. Previous studies have observed vascular lesions in 30\%-80\% of patients with ischemic stroke. ${ }^{4,11,12,15}$ To translate this work to the general population, these discrepancies must be addressed.

The time interval from stroke onset to hospital arrival is considered first. Two studies conducted during the hyperacute stage (3-6 hours) reported that approximately $80 \%$ of patients had vascular occlusions on MRA and digital subtraction arteriography. ${ }^{12,21}$ Other studies that included patients 1 day after stroke observed SYSO in approximately $30 \%$ of patients. ${ }^{11,15}$ Recanalization of the steno-occluded vessels during the hyperacute stage could explain the discrepancy between these studies, and rapid examination of the cerebral vasculature could be helpful in the detection of steno-occlusion. ${ }^{16}$

The lack of a consensus on the definition of SYSO and differences in diagnostic modalities also affect the detection rate of SYSO. In this work, we included more than a mild degree of symptomatic stenosis and occlusion detected by MRA. However, previous studies using Doppler ultrasonography detected $30 \%$ of symptomatic vessels with more than $50 \%$ stenosis or occlusion. ${ }^{11}$

Consistent with previous studies that showed the effect of ethnicity, ${ }^{22}$ involvement of the intracranial artery was observed in $72 \%$ of patients in this study; this is similar to the findings of a Chinese study $y^{23}$ and higher than that seen in Spanish $(56.8 \%)^{11}$ and German $(37.6 \%)^{15}$ studies. Interestingly, the ratio of stroke subtype in this study was similar to that in the German study (Table 1). After examining the risk factors for stroke, the most frequent cause of stroke in patients with SYSO was large artery disease, followed by cardioembolic stroke.

Approximately $30 \%$ of patients with SYSO (19.3\% of all sub- 
jects in the study) had 2 or more symptomatic vascular lesions. Compared with previous findings of multiple vascular lesions in 4\%-18\% of patients, an MRA-based study may detect a high proportion of cases of multiple SYSOs. In support of this, a previous study reported that $30 \%$ of patients with acute ischemic stroke had multiple ischemic lesions on diffusion-weighted magnetic resonance imaging. ${ }^{24}$ We found no significant difference in demographic profiles or clinical characteristics between patients with single and multiple SYSOs. The most frequent stroke subtype in patients with multiple SYSOs was large artery disease rather than cardioembolic stroke.

In this study, SYSO at acute stages of ischemic stroke increased the relative risk of a poor outcome to $77 \%$. This finding suggests that clinicians need to pay more attention to SYSO in addition to previously noted predictors such as lesion volume, ${ }^{25}$ stroke severity, ${ }^{26}$ and administration of various agents at acute stages. ${ }^{5,6}$

We investigated the effects of the characteristics of SYSO, including quantity, location, and severity, on the severity of outcome. The location of SYSO in terms of anterior versus posterior circulation and individual arterial location did not affect the stroke outcome. This finding was consistent with the post-hoc analysis of the Warfarin versus Aspirin for Symptomatic Intracranial Disease trial, which reported that the location of intracranial stenosis was not associated with recurrence of ischemic stroke. ${ }^{13}$ The individual arterial location also did not have a statistically significant effect on outcome. However, the results of the effect of individual arteries on stroke outcome should be interpreted with caution. Highly correlated relationships between the individual artery site and the baseline severity of stroke as previously described ${ }^{14,21}$ might have diminished the statistical power.

The severity of SYSO varied, depending on the individual artery. In the MCA and EICA, moderate to severe stenosis and occlusion were frequently observed and the proportion of poor outcomes was related to the severity of SYSO. In contrast, the BA and VA did not show this relationship. However, the effect of severity based on the arterial location did not show a significant interaction. We observed a relationship between severity and the risk of a poor outcome when considering a single SYSO. Finally, multiple SYSOs were associated with a worse outcome than a single SYSO even though there were few differences in the clinical characteristics. Further research to explain these results is warranted.

This study had several limitations. First, it is difficult to assess the generalizability of this study because MRA was used exclusively. However, MRA is used in $96.8 \%$ of patients with ischemic stroke, which may resolve this limitation to some degree.
Second, there is the possibility of a selection bias in this study because of limited hospital participation. However, the large number of patients nationwide from scattered community-based hospitals is believed to provide enough power to detect relatively large effects of SYSO on stroke outcome. Lastly, stroke outcome was observed at discharge or transfer, which would prohibit elucidating its effect on 3-month stroke outcome.

In conclusion, we showed that approximately $60 \%$ of patients with acute ischemic stroke have SYSO and that many of these patients have multiple vessels involved. SYSO is an independent risk factor for poor clinical outcome, and this risk is affected by the number and severity of SYSOs.

\section{References}

1. Derex L, Nighoghossian N, Hermier M, Adeleine P, Froment JC, Trouillas P. Early detection of cerebral arterial occlusion on magnetic resonance angiography: predictive value of the baseline NIHSS score and impact on neurological outcome. Cerebrovasc Dis 2002;13:225-229.

2. Chimowitz MI, Lynn MJ, Howlett-Smith H, Stern BJ, Hertzberg VS, Frankel MR, et al. Comparison of warfarin and aspirin for symptomatic intracranial arterial stenosis. N Engl J Med 2005;352:1305-1316.

3. Paciaroni M, Caso V, Venti M, Milia P, Kappelle LJ, Silvestrelli $\mathrm{G}$, et al. Outcome in patients with stroke associated with internal carotid artery occlusion. Cerebrovasc Dis 2005;20:108-113.

4. Wong KS, Li H. Long-term mortality and recurrent stroke risk among Chinese stroke patients with predominant intracranial atherosclerosis. Stroke 2003;34:2361-2366.

5. Tissue plasminogen activator for acute ischemic stroke. The national institute of neurological disorders and stroke rt-pa stroke study group. N Engl J Med 1995;333:1581-1587.

6. Furlan A, Higashida R, Wechsler L, Gent M, Rowley H, Kase $\mathrm{C}$, et al. Intra-arterial prourokinase for acute ischemic stroke. The PROACT II study: a randomized controlled trial. Prolyse in Acute Cerebral Thromboembolism. JAMA 1999;282:20032011.

7. Eckstein HH, Schumacher H, Klemm K, Laubach H, Kraus T, Ringleb P, et al. Emergency carotid endarterectomy. Cerebrovasc Dis 1999;9:270-281.

8. Bose A, Hartmann M, Henkes H, Liu HM, Teng MM, Szikora I, et al. A novel, self-expanding, nitinol stent in medically refractory intracranial atherosclerotic stenoses: the wingspan study. Stroke 2007;38:1531-1537.

9. Imai K, Mori T, Izumoto H, Watanabe M, Majima K. Emergency carotid artery stent placement in patients with acute ischemic stroke. AJNR Am J Neuroradiol 2005;26:1249-1258. 
10. Jahromi BS, Tummala RP, Yamamoto J, Levy EI, Siddiqui AH, Hopkins LN. Early carotid stenting for symptomatic stenosis and intraluminal thrombus presenting with stroke. Neurology 2008;71:1831-1833.

11. Ois A, Cuadrado-Godia E, Jimenez-Conde J, Gomis M, Rodriguez-Campello A, Martinez-Rodriguez JE, et al. Early arterial study in the prediction of mortality after acute ischemic stroke. Stroke 2007;38:2085-2089.

12. Schellinger PD, Fiebach JB, Jansen O, Ringleb PA, Mohr A, Steiner T, et al. Stroke magnetic resonance imaging within 6 hours after onset of hyperacute cerebral ischemia. Ann Neurol 2001;49:460-469.

13. Kasner SE, Chimowitz MI, Lynn MJ, Howlett-Smith H, Stern BJ, Hertzberg VS, et al. Predictors of ischemic stroke in the territory of a symptomatic intracranial arterial stenosis. Circulation 2006; 113:555-563.

14. Saqqur M, Uchino K, Demchuk AM, Molina CA, Garami Z, Calleja S, et al. Site of arterial occlusion identified by transcranial doppler predicts the response to intravenous thrombolysis for stroke. Stroke 2007;38:948-954.

15. Weimar C, Goertler M, Harms L, Diener HC. Distribution and outcome of symptomatic stenoses and occlusions in patients with acute cerebral ischemia. Arch Neurol 2006;63:1287-1291.

16. Meves SH, Muhs A, Federlein J, Buttner T, Przuntek H, Postert T. Recanalization of acute symptomatic occlusions of the internal carotid artery. J Neurol 2002;249:188-192.

17. Alexandrov AV, Demchuk AM, Wein TH, Grotta JC. Yield of transcranial doppler in acute cerebral ischemia. Stroke 1999;30: 1604-1609.

18. Wildermuth S, Knauth M, Brandt T, Winter R, Sartor K, Hacke W. Role of CT angiography in patient selection for thrombo- lytic therapy in acute hemispheric stroke. Stroke 1998;29:935938.

19. Hong KS, Bang OY, Kang DW, Yu KH, Bae HJ, Lee JS, et. al. Stroke Statistics in Korea: part I. Epidemiology and Risk Factors: a Report from the Korean Stroke Society and Clinical Research Center for Stroke. Journal of Stroke 2013;15:2-20.

20. Oelerich M, Lentschig MG, Zunker P, Reimer P, Rummeny EJ, Schuierer G. Intracranial vascular stenosis and occlusion: comparison of 3D time-of-flight and 3D phase-contrast $\mathrm{MR}$ angiography. Neuroradiology 1998;40:567-573.

21. Fischer U, Arnold M, Nedeltchev K, Brekenfeld C, Ballinari P, Remonda L, et al. NIHSS score and arteriographic findings in acute ischemic stroke. Stroke 2005;36:2121-2125.

22. Sacco RL, Kargman DE, Gu Q, Zamanillo MC. Race-ethnicity and determinants of intracranial atherosclerotic cerebral infarction. The Northern Manhattan Stroke Study. Stroke 1995;26: 14-20.

23. Wong KS, Li H, Chan YL, Ahuja A, Lam WW, Wong A, et al. Use of transcranial doppler ultrasound to predict outcome in patients with intracranial large-artery occlusive disease. Stroke 2000;31:2641-2647.

24. Roh JK, Kang DW, Lee SH, Yoon BW, Chang KH. Significance of acute multiple brain infarction on diffusion-weighted imaging. Stroke 2000;31:688-694.

25. Thijs VN, Lansberg MG, Beaulieu C, Marks MP, Moseley ME, Albers GW. Is early ischemic lesion volume on diffusion-weighted imaging an independent predictor of stroke outcome? A multivariable analysis. Stroke 2000;31:2597-2602.

26. Appelros P, Nydevik I, Viitanen M. Poor outcome after firstever stroke: predictors for death, dependency, and recurrent stroke within the first year. Stroke 2003;34:122-126. 
Supplementary Table 1. Baseline characteristics of study subjects and comparisons by functional outcome at discharge

\begin{tabular}{lccr}
\hline Variables & $\begin{array}{c}\text { Good outcome } \\
\mathrm{N}=1,656\end{array}$ & $\begin{array}{c}\text { Poor outcome } \\
\mathrm{N}=1,401\end{array}$ & $P$ \\
\hline Age & $64.8 \pm 12.4$ & $70.2 \pm 12.0$ & $<0.001$ \\
Male & $1,061(64.1 \%)$ & $740(52.8 \%)$ & $<0.001$ \\
Hypertension & $1,084(65.5 \%)$ & $931(66.5 \%)$ & 0.564 \\
Diabetes & $444(26.8 \%)$ & $468(33.4 \%)$ & $<0.001$ \\
Dyslipidemia & $368(22.2 \%)$ & $288(43.9 \%)$ & 0.264 \\
high risk cardioembolism & $284(17.1 \%)$ & $389(27.8 \%)$ & $<0.001$ \\
Smoking & $684(41.3 \%)$ & $502(35.8 \%)$ & 0.002 \\
History of stroke & $297(17.9 \%)$ & $358(25.6 \%)$ & $<0.001$ \\
Initial NIHSS, median (IOR) & $3(1-6)$ & $6(2-14)$ & $<0.001$ \\
Stroke subtype & & & $<0.001$ \\
SVD & $463(70.7 \%)$ & $192(29.3 \%)$ & \\
LAD & $531(49.1 \%)$ & $551(50.9 \%)$ & \\
CE & $320(42.2 \%)$ & $438(57.8 \%)$ & \\
Thrombolysis & $188(11.4 \%)$ & $273(19.5 \%)$ & $<0.001$ \\
IA & $31(1.9 \%)$ & $95(6.8 \%)$ & $<0.001$ \\
IV+IA & $22(1.3 \%)$ & $52(3.7 \%)$ & $<0.001$ \\
SBP, initial & $149.3 \pm 27.1$ & $149.3 \pm 28.2$ & 0.945 \\
DBP, initial & $86.4 \pm 14.6$ & $86.5 \pm 16.1$ & 0.761 \\
\hline
\end{tabular}

Values are presented as numbers (percentage) except for special comment variables. Functional outcome at discharge was adjudicated as good (mRS 0-2) and poor (mRS 3 or more). Abbreviations are expressed as IQR is interquartile range; CE for cardioembolism; NIHSS for National Institute of Health Stroke Scale; IA for intraarterial thrombolysis; and IA+IV for intraarterial and intravenous thrombolysis.
Supplementary Table 2. Multivariable logistic model to evaluate the effect of SYSO to the stroke outcome

\begin{tabular}{lcc}
\hline Variables & Adjusted odds ratio & 95\% confidence interval \\
\hline Age & 1.03 & $1.02-1.03$ \\
Male & 0.66 & $0.53-0.82$ \\
History of stroke & 1.30 & $1.04-1.61$ \\
Baseline NIHSS score & & \\
1st quartile & 1.00 (reference) & \\
2nd quartile & 4.24 & $3.24-5.54$ \\
3rd quartile & 12.82 & $9.89-16.58$ \\
4th quartile & 48.98 & $35.54-67.52$ \\
Diabetes mellitus & 1.54 & $1.27-1.88$ \\
High risk of CE & 0.77 & $0.61-0.97$ \\
Smoking & 1.27 & $1.01-1.58$ \\
Thrombolysis & 0.52 & $0.40-0.67$ \\
SYSO & 1.77 & $1.46-2.15$ \\
\hline
\end{tabular}

Outcome was poor functional outcome at discharge (modified Rankin Disability Score $\geq 3$ ). Abbreviations are expressed as CE for cardioembolism; NIHSS for National Institute of Health Stroke Scale. 